\title{
The Influence of Linkages, Feedback Mechanisms, and Caregivers' Mobility on Immunization Follow-Up Visits in Lideta Sub-City of Addis Ababa, Ethiopia: A Qualitative Study
}

Thewodros Zewde Shay ( $\square$ Thewodroszewde40@gmail.com )

MERQ Consulting P.L.C https://orcid.org/0000-0001-8696-5089

Alula Meresa Teklu

MERQ Consulting PLC

Diriba Bedada Hunde

MERQ Consultancy P.L.C

Yoseph Tsehaye Sultan

Lideta sub-city Health Buraeu

\section{BMC Supplements Reviewed}

Keywords: linkages, feedback mechanism, follow-up, transferal process, mobility, transferred out

Posted Date: February 5th, 2021

DOl: https://doi.org/10.21203/rs.3.rs-172939/v1

License: (c) (1) This work is licensed under a Creative Commons Attribution 4.0 International License.

Read Full License

Version of Record: A version of this preprint was published at Health Research Policy and Systems on August 11th, 2021. See the published version at https://doi.org/10.1186/s12961-021-00690-5. 


\section{Abstract}

\section{Background}

Losing children to follow-up is one of the major barriers identified in Ethiopia's immunization program. In many urban slum areas like Lideta sub-city, Addis Ababa, several demand- and supply-side factors affect the follow-up visits for routine immunization services, so this study aimed to explore the influence of linkages, a feedback mechanism, and caregivers' mobility on immunization follow-up visits.

\section{Methods}

The study team employed a qualitative method and conducted 30 in-depth interviews with caregivers, 26 interviews with key informants, and five focus group discussions with health officials and decisionmakers. A deductive content and thematic analysis was carried out by importing the transcripts into OpenCodes, applying the a priori codes, and identifying new codes and themes.

\section{Results}

The linkages among health facilities included those from hospitals to health centers, from hospitals to hospitals, and from health centers to health centers within and outside of the sub-city. Using these linkages, most vaccinators transfer caregivers without providing multi-dose vial (MDV) vaccines, mainly BCG and MCV, 'to minimize wastage' and thus successfully reduce vaccine wastage rates; yet most caregivers wasted their time, energy, and money traveling from one health facility to another. Despite some efforts to transfer caregivers using 'transferal slips' and informal phone calls to vaccinators' friends, unfortunately, there was no formally established system for getting feedback about the arrival of caregivers and continuation of the follow-up visits. Overall, the transfer process lacked uniformity, used various approaches, and was not systematic.

\section{Conclusions}

Transferal of caregivers for the sake of minimizing wastage of MDV vaccines without checking the vaccination schedules of the receiving health facilities, using various informal types of tools and approaches, along with a poor follow-up and feedback system were major identified challenges, which cost caregivers extra money, energy, and time in getting timely immunization services. Therefore, the Federal Ministry of Health should strengthen the linkages among facilities, ensure the establishment of formal communications by developing guidelines and standardized tools-transferal slips and approaches-and initiate a fast feedback provision system using SMS text messages.

\section{Background}

In Africa, more than 30 million children under 5 suffer from vaccine-preventable diseases (VPDs) every year. Of these, over half a million children die from VPDs annually due to limited access to immunization services, accounting for $58 \%$ of all global deaths among under-5 children due to VPDs [1]. In Ethiopia, 
about $3 \%$ of children under 5 die due to measles [2]. Thus, almost every public health facility in the country provides 12 antigens, including the measles-rubella vaccine (MRV) and others, such as the bacillus Calmette-Guérin (BCG), oral polio vaccine (OPV), pentavalent vaccine (diphtheria, pertussis, tetanus, hepatitis $\mathrm{B}$, and Haemophilus influenzae B), pneumococcal conjugate vaccine (PCV), inactivated poliovirus vaccine (IPV), and rotavirus vaccine (RV), through the routine immunization (RI) programs targeting measles and the other major VPDs during childhood [3].

Concurrently, the country has a well-structured public health care system around the concept of a "health network model," which is composed of three tiers of vertically linked levels-primary, secondary, and tertiary-each with defined catchment populations. The primary level in rural settings includes primary health care units (PHCUs), defined as five satellite health posts with a health center and primary/district hospital. In the urban setting, the health center is the primary entry point to the health system. Secondarylevel health care includes general hospitals (zonal/regional), and tertiary-level health care includes tertiary hospitals (central/referral) [4-6]. Under vaccination service provision, demand for vaccines and vaccination is a complex concept that is not external to supply systems but rather encompasses the interaction between human behaviors and system structure and dynamics [7]. Correspondingly, the country reaches targeted groups through the existing system, and "transferal" of children for vaccination (defined in this study as "informing a caregiver of a child to go to any level of health facility to get vaccines for her child") is common among health facilities. A child can be transferred across levels of facilities, for example from a higher-level hospital to a lower-level hospital or health center (i.e., a vertical transfer). However, a child can also be transferred from one health center to another health center of the same level (i.e., horizontally).

Additionally, there are various factors that can influence the service provision and transferal process within the structure, and in many urban slum areas, where housing and living conditions are exceptionally poor [8], one of the major problems for access to and utilization of services is losing children before follow-up visits. This means that once some caregivers have accessed and received routine immunization services for their children from one health facility at a given time, they fail to show up for their subsequent visits at the same facility based on their appointment date. On top of that, improvement of urban immunization programs requires collaboration among health facilities [9]; the lack of wellfunctioning linkages and strong feedback mechanisms between private and public health facilities and among public health facilities, the absence of a formal communication system among district health offices, and the increased number of self-referrals for many reasons are some of the other causes of losing children to follow-up.

Despite the existence of various demand- and supply-side factors that may influence immunization service uptake, little is known about how these factors are playing out in urban slum areas in Ethiopia. Hence, this study explores how the functionality of linkages and feedback mechanisms among health facilities and the mobility of caregivers affect follow-up visits in routine immunization services. It assesses the steps and situations that the transferred-out caregivers encounter in the process of transferring from one health facility to another and examines the existence of functional linkages among 
health facilities and the availability of a feedback system, active collaboration and formalized communication between health facilities, the availability of formal and EPI program-specific procedures and tools for linking caregivers between health facilities, the quality of the accountability for providers' performance and supportive supervision to improve the linkage system, and how the government can provide support for these linkages.

Subsequently, recommendations are made regarding feasible and innovative strategies to improve bilateral communications and active collaboration among health facilities that provide routine immunization services benefiting targeted children so caregivers can access these services easily and thereby reduce the number of patients lost to follow-up.

\section{Methods}

Study Setting: The study was conducted in Lideta sub-city, Addis Ababa, Ethiopia, which was purposely selected, as it has several slum areas; poor vaccination coverage of $31.5 \%$ BCG, 44.7\% Penta-1, 47.6\% Penta-3, and $42.7 \%$ MCV; and high loss to follow-ups due to construction of condominium apartments and displacement of residents. The sub-city has an area of 918.4 square $\mathrm{km}, 10$ districts, 60,196 households, a total population of 246,803 , six hospitals, six health centers, and 52 private clinics. All the health centers and four of the hospitals provide routine vaccination services.

Study Design: The study team employed a qualitative research approach to answer the main research question: How do linkages, feedback mechanisms, and caregivers' mobility influence immunization follow-up visits?

Sampling: The first 30 caregivers were conveniently selected considering their availability for follow-up. Meanwhile, 26 key informants and five focus group discussants were purposely selected from all levels considering their roles and contributions in the EPI program. Except three key informants, the rest participated in the Focus Group Discussion (FGDs) (Table-1). 


\begin{tabular}{|c|c|c|c|c|}
\hline Methods & Respondents & Male & Female & Total \\
\hline IDIs & Caregivers & 0 & 30 & 30 \\
\hline \multirow[t]{6}{*}{ KIIs } & UHEWs & 0 & 3 & 3 \\
\hline & Medical directors (HCs) & 4 & 1 & 5 \\
\hline & EPI focal (HCs + Hospitals) & 5 & 7 & 12 \\
\hline & Coordinators/Supervisors (District) & 3 & 1 & 4 \\
\hline & Coordinators/Supervisors (Sub-City) & 1 & 1 & 2 \\
\hline & Total & 13 & 13 & 26 \\
\hline \multirow[t]{6}{*}{ FGDs } & Medical directors (HCs) & 6 & 0 & 6 \\
\hline & EPI focal (HCs) & 0 & 7 & 7 \\
\hline & UHEWs & 0 & 6 & 6 \\
\hline & EPI focal (Hospitals) & 0 & 6 & 6 \\
\hline & Coordinators/Supervisors (Districts + Sub-city) & 4 & 4 & 8 \\
\hline & Total & 10 & 23 & 33 \\
\hline
\end{tabular}

Data Collection: The data collection and transcription activities took a total of 4 months (Aug-Nov 2017). In-depth interviews (IDIs) were conducted at health facilities, while key informant interviews (KIIs) were done at workplaces and FGDs at meeting halls. All contacted participants agreed to participate; hence, all interviews and discussions were conducted in Amharic using pre-tested guides in quiet places at a convenient time. MPH degree-holder moderators and note-takers carried out the IDIs and KIIs, and the authors led the FGDs. The note-takers recorded the data using digital tape recorders and notebooks. On average, the IDIs were finished within 45 minutes, while the KIls and FGDs took 90 minutes each. The study team ensured data saturation by listening to and discussing tape-recorded data on a weekly basis, repeating $5 \%$ of IDIs and KIls and returning transcripts to key informants and discussants for further validation and correction.

Data analysis: A deductive content and thematic analysis approach was applied. First, an initial set of codes were generated and aligned with the research questions by the authors. As the initial sets of codes consisted only of major categories, the code categories were further refined, and sub-codes were developed. Then, the analysis took place by importing the transcripts into OpenCodes (QDR: https://doi.org/10.5064/F6HMZBEL) and applying the a priori codes and new codes.

Ethical Considerations: The Scientific and Ethics Review Committee (WHO ERC) and local IRB approved the protocol. No subjects were interviewed without providing informed consent. 


\section{Results}

The findings are presented in three broad sections: existing linkages, transferal processes and caregivers' mobility, and feedback mechanisms with major barriers.

\section{Existing Linkages}

The linkages among health facilities included those from hospitals (government and private) to health centers, from hospitals to hospitals, and from health centers to health centers. The linkages focused on what exists within health facilities found within or outside of the sub-city. These facilities built their linkages with facilities that were nearby and easily accessible. Within a health facility, the first linkages were initiated between the delivery service unit and the EPI unit, as newborn babies required the first vaccine (BCG) at birth. In linking caregivers from the community level with health centers, UHEWs were the key players, with the responsibilities of identifying and transferring those children who hadn't attended vaccination at all or those children who had defaulted from their vaccination schedules.

Transferal Processes and Caregivers' Mobility

\section{Multi-Dose Vial Policy (MDVP) for BCG vs. Inconvenient BCG Vaccination Sessions at Health Facilities}

Even though most vaccinators knew the national BCG vaccination schedule was at birth, MDVP became the main reason for their transferal of caregivers. That is, most of the caregivers were not able to get the BCG vaccine for their children immediately following delivery, as they were transferred out by nurses or by themselves (self-transferal) because vaccinators open a BCG vaccine only when they get enough children, as they strive to reduce BCG vaccine wastage.

"Just post-delivery on Wednesday at [Hospital], the nurses told me that I could either bring my baby on Thursday [BCG opening day] for vaccination or get the service from a nearby health center. So, I preferred to come here [Health Center] because it is close to my house. On the next day, my baby was vaccinated." (A caregiver, female)

The vaccinators advised caregivers either to go immediately post-delivery discharge or on the 45th day after delivery. Differently, some other vaccinators preferred taking the phone numbers of caregivers and calling them when the required number of children attended.

"The nurses asked me which health facility is nearest to me, and I told them the name of the Health Center. Then they gave me a piece of paper and told me that I could continue the follow-up on the 45th day after birth." (A caregiver, female)

\section{Blind Transferal Due to Lack of Formal Communication and Information}


Besides the efforts of some vaccinators who knew the schedules of a few nearby health centers by calling nurses they knew on their mobile phones, most of them still had problems getting the exact dates and times when BCG and MCV vaccines could be opened at the other health facilities. As a result, they transferred caregivers without having clear information, so the caregivers had to pay unnecessary transport costs and felt that their time and energy were wasted.

"When a caregiver arrives on a date on which BCG and MCV are not given, we will either appoint the caregivers to another time or transfer them to another nearby health facility." (A public health officer, male)

\section{Absence of Standardized Tools and Procedures in the Transferal Process}

When some vaccinators transferred out caregivers, they advised them to take their child's vaccination yellow card or provided transferal slips or a simple piece of paper, while others tried communicating with the facilities by phone before they transferred caregivers to them. Meanwhile, UHEWs used a medical referral form.

"We transfer caregivers out to the health facility where they need to go, and then we record that in our registration book. We do not record the name of the facility where the caregivers want to continue on the transfer paper." (A vaccinator, female)

A couple of vaccinators expressed the challenge of providing vaccination services to those caregivers who visited them without any document, as they couldn't clearly tell which vaccines their children had already received. Some caregivers counted the eye ointment and Vitamin K injection that were provided to their children during delivery as vaccines.

"Some caregivers come without any evidence and tell us, 'He [their child] has been vaccinated,' but they do not know what vaccine he received. Sometimes, they come with cards that indicate his weight and tell us, 'He received a vaccine in another facility, and that facility informed us to continue his vaccination here.' ... Sometimes children come from hospitals with a small card that indicates SVD delivery. ... Mothers who come from kebele do not bring anything for evidence, and they debate with us, they say, 'He already received this,' or 'He did not receive this'." (A vaccinator, male)

\section{Checking Caregivers' Mobility}

Most vaccinators asked caregivers for their residence addresses, whether they needed that day's vaccination or would continue their follow-up at their health facility, and whether they had a plan to change their home or stay in same house, and they also checked the appointment dates.

"When mothers come to our health center, first we ask them their reason for coming here. If their intention is to get today's vaccine, we don't provide the service. We just send them back, but if they want to 
continue in our health center due to change of residence or other reasons, we accept and vaccinate their children." (A vaccinator, female)

Feedback Mechanisms

\section{Absence of Established Formal Feedback System}

Most of the EPI focal persons expressed that there was no system in place to follow the outcomes of transferred-out children. Only a few self-motivated vaccinators would call caregivers and ask them if they had gone to the receiver health facilities. Nonetheless, UHEWs had a chance to learn whether a transferred-out child had reached the health center and received the required vaccine or not during their house-to-house visits.

"I think there are some gaps. We transfer out a lot of children, but there is no way for us to get feedback. But what we hope is that the caregivers will visit the health facilities because we inform them a lot about how beneficial the immunizations are for their babies." (A vaccinator, female)

\section{Discussion}

Linkages were first initiated between two units within a hospital or health center--the delivery service unit and EPI unit-when a caregiver gave birth, as a newborn baby requires the first vaccine (BCG) at birth. Then, the linkages moved to only among the EPI units of health facilities in the three-tier health system: between hospitals and hospitals, hospitals and health centers, health centers and health centers, and health centers and the community through UHEWs within or outside of the sub-city. Through the path of the linkages, the transferal process was established, and caregivers moved from initiating health facilities to receiving facilities, which were closer and more easily accessible to them. Almost all vaccinators transferred out caregivers who needed multi-dose vial (MDV) vaccines, particularly BCG and MCV.

Even though newborn vaccination (BCG, OPV-0, and $\mathrm{HepB}$ ) is a critical parameter for evaluating the overall performance of immunization programs [10], most vaccinators transferred out caregivers without providing BCG "to minimize BCG wastage" -in adherence to the Multi Dose Vial Policy (MDVP), which permits certain vaccines to be stored for up to 28 days after opening, and any other actual or perceived thresholds for opening a vial [11]. As a result, they reported successful reduction of vaccine wastage rates as strength; yet most caregivers wasted their time, energy, and money by traveling from one health facility to another. Similarly, caregivers faced the same challenges in getting MCV, as a change in the number of doses per container affects operational costs, timely coverage, safety, product costs, and policy [12].

In achieving maximum utilization of every dose, vaccinators need to be proactive with communication to ensure optimal attendance and timely vaccination of every child [13]; but even though communication activities are a crucial element of immunization programs [14], none of the vaccinators knew the vaccination schedules of receiving health facilities through formal communication channels. Meanwhile, use of phone contacts to follow up is a feasible and cost-effective method for tracking children who have missed out [15] that has been successfully used by community health workers in some urban settings 
[16]. Despite some efforts to transfer caregivers using "transferal slips" or pieces of paper or through informal phone calls to vaccinators' friends, unfortunately, there was no formally established system for transferring caregivers and getting feedback. Besides, there was no way for the receiver health facility to notify the first health facility from which the caregivers had been transferred about their arrival.

Nonetheless, many caregivers became dissatisfied and did not continue follow-up due to travel to several health facilities and reappointments.

The main strengths of the study include the transferal processes observed among various types of health facilities with different characteristics. Although demand for vaccines and access to immunization services is a complex concept that is not external to supply systems $[15,17]$, the study covered caregivers from the demand side and UHEWs, vaccinators, medical directors, supervisors, EPI focal persons, and managers from the supply side, so the behavior of both service providers and users was properly observed. Considering the more pervasive and common patterns of schedule non-completion among poorer urban mothers [18-21] and the identified gaps, the authors make the following appropriate recommendations: The Federal Ministry of Health (FMOH) should develop a protocol with standardized transferal tools to facilitate the transferal process and ensure accountability; support the appropriate implementation of MDVP; initiate a formal feedback provision system using SMS text messages; and build the capacity of vaccinators, EPI focal persons, supervisors, and managers to ensure formal transferal processes with feedback.

Potentially, the study's findings have limited generalizability to other places because the linkages between health centers and health posts were not observed, as there are no health posts overall in the capital. Therefore, the linkage and transferal system between health centers and health posts should be investigated in the rural and pastoralist regions of the country. On the other hand, it was not possible to get caregivers who travelled to places far outside the capital, so the sample might not be representative. Currently, there are emerging productions of new multi-dose vial vaccines like PCV; user countries should conduct similar studies to assess the linkages and transferal processes in accessing BCG, measles, and PCV vaccines for children.

\section{Conclusion}

Fragile linkages, transferal processes, the feedback system, and caregivers' mobility affect follow-up visits for immunization services. Transferal of caregivers for the sake of minimizing MDV vaccine wastage without checking the vaccination schedules of receiving health facilities, using various types of tools and approaches, along with a poor follow-up and feedback system cost caregivers extra time, energy, and money for transport to get timely services by moving from one health facility to another. Hence, FMOH should ensure formal communication and transferal processes by developing guidelines, standardized tools, and procedures; initiating feedback systems using SMS text messages; and building vaccinators' capacities.

\section{Abbreviations}


BCG Bacillus Calmette-Guérin

EPI Expanded Program of Immunization

EPHI Ethiopian Public Health Institute

FGD Focus Group Discussion

FMOH Federal Ministry of Health

IDI In-Depth Interview

IPV Inactivated Poliovirus Vaccine

KII Key Informant Interview

OPV Oral Polio Vaccine

MCV Measles Containing Vaccines

MRV Measles-Rubella Vaccine

MDV Multi-Dose Vial

MDVP Multi-Dose Vial Policy

PCV Pneumococcal Conjugate Vaccine

PHCUs Primary Health Care Units

RV Rotavirus Vaccine

RI Routine Immunization

SMS Short Message Service

SVD Self Vaginal Delivery

UHEWs Urban Health Extension Workers

VPD Vaccine-Preventable Diseases

\section{Declarations}

Ethics approval and consent to participate: The Research Ethics Review Committee (WHO ERC) approved the protocol (reference no. ERC.0002843). Besides, the Scientific and Ethics Review Committee under the Ethiopian Public Health Institute approved the protocol. 
Participants were given a copy (unsigned) of the informed consent statement that included contact information for research team members. The informed consent forms were written at a readability level appropriate for the target populations. Additionally, the consent statement included the purpose of the research; clarified the expected duration of their participation; how the research benefited the children, the caregivers, and the community; there were no costs for participating; the caregiver's participation was voluntary and that refusal to participate had no consequences.

No subjects were interviewed without providing informed consent. Written informed consent was obtained prior to the interview, and consent was verified orally at the beginning of the interview. The consent form was read aloud by interviewers to the caregivers, after which the caregivers were asked if they understood and agreed to participate. The caregivers and the person obtaining consent/assent signed the form. Caregivers unable or unwilling to sign their name were made a mark, and a witness of the consent/assent procedures were signed on their behalf.

Consent for Publication: the plan for sharing findings through publications and conferences had been incorporated within the informed consent and the caregivers and other key informants of the study agreed to publish any of their given responses without indicating their names.

Availability of data and materials: The datasets used and analyzed during the current study are available from the corresponding author upon reasonable request.

Competing interests: The authors declare that they have no competing interests.

Funding: The full amount of the payments was made by GAVI for WHO/Alliance for Health Policy and Systems Research (AHPSR), DELIR Initiative. Meanwhile, the consortium members-GAVI, UNICEF and AHPSR provided technical support in refining the research proposal to feasible protocol, monitoring the process at all levels of the implementation, reviewing the final reports and disseminating the major findings. They also developed an instructional guide for developing manuscripts and some relevant reporting guidelines. Further, they designated two internal reviewers from the Alliance and UNICEF to review the manuscript and provide with feedback. The guide and feedback from the internal review has been used to revise and further develop the manuscript prior to submission to BMC supplement.

Costs for Publication: publication costs are funded by WHO, Alliance for Health Policy and System Research (AHPSR), Decision-Maker Led Implementation Research (DELIR) initiative.

Authors' contributions: Y.T.; Collected secondary data and other health profiles from Lideta Sub-City Health Bureau and fed for protocol development. T.Z., D.B.; Developed the protocol and required tools, submitted for WHO ERC and local IRB and addressed the comments given by review committee. T.Z.; Followed the WHO ERC process. T.Z., A.T.; Facilitated the local IRB processes. T.Z., D.B., A.T., Y.T.; Conceptualized the study, provided pre-data collection trainings for facilitators of the IDIs, KIls and FGDs, oversaw data collection at field level, monitored the transcription process, supported the data analysis and interpretation of the findings. T.Z., D.B., A.T., Y.T.; Wrote the main report of the study and contributed 
to the write-up of the draft and final versions of the manuscript. T.Z., D.B., A.T., Y.T.; Made all the major and minor changes in the manuscript. On the other hand, all authors have read and approved the final manuscript to publish.

Acknowledgements: The authors would like to acknowledge the hard work, dedication, and lively spirit of collaboration from everyone involved in completing this decision-maker-led research to address highpriority immunization issues. We acknowledge the intellectual contributions of the vaccinators, medical directors, EPI focal persons, coordinators, supervisors, UHEWs, and caregivers who participated in indepth interviews, key informant interviews, and focus group discussions. We also wish to sincerely express our gratitude and indebtedness to the core group of experts and research assistants that made the field assessment a success. We thank Wogayehu Tadele, Munit Legesse, Yosef Sultan, Sintayehu Abate, and Ibrahim Kedir for their technical guidance during the planning of the assessment and in selecting interviewees and discussants and for effectively coordinating and facilitating fieldwork. We also extend our gratitude to the MERQ consultancy office's admin staffs for their coordination, and to the study respondents, who willingly lent their time to the study. Finally, we wish to acknowledge the following partners: GAVI, WHO/Alliance for Health Policy and Systems Research, UNICEF for first protocol review and approval and also overall technical advice in the report write-up, manuscript preparation and revision; $\mathrm{EPHI}$ for reviewing the protocol and for their observations during trainings and data collection processes; the $\mathrm{FMOH}$, Addis Ababa health bureau, and Lideta sub-city health bureau for accepting the EPHI approval and permission; and district health offices and health facilities for assigning guides and arranging interviews.

\section{References}

1. World Health Organization. Business case for WHO immunization activities on the African continent 2018-2030.

2. FMOH document. Health Sector Transformation Plan (HSTP) of Ethiopia 2016-2020. Oct 2015. Addis Ababa, Ethiopia.

3. FMOH document. Comprehensive Multi-Year Plan (CMYP) for Immunization of Ethiopia 2016-2020. April 2015. Addis Ababa, Ethiopia.

4. FMOH document. National Newborn and Child Survival Strategy 2016-2020. June 2015. Addis Ababa, Ethiopia.

5. FMOH document. Strategic Framework for Referral and Linkages between HCT and Chronic HIV Care Services in Ethiopia. Aug 2009. Addis Ababa, Ethiopia.

6. FMOH document. Public Private Partnership in Health-Strategic Framework for Ethiopia. June 2013.

7. Mokaya E, Mugoya I, Raburu J, Shimp L. Use of cellular phone contacts to increase return rates for immunization services in Kenya. The Pan African Medical Journal. 2017;28.

8. Atkinson SJ, Cheyne J. Immunization in urban areas: Issues and strategies. Bulletin of the World Health Organization. 1994;72(2):183. 
9. World Health Organization. WHO policy statement: Multi-dose vial policy (MDVP): Handling of multidose vaccine vials after opening.

10. Taneja G, Mentey VK, Jain M, Sagar KS, Tripathi B, Favin M, Steinglass R. Institutionalizing early vaccination of newborns delivered at government health facilities: Experiences from India. International Journal of Medical Research and Review. 2015 Jun 1;3(5):521-7.

11. Cutts FT. Strategies to improve immunization services in urban Africa. Bulletin of the World Health Organization. 1991;69(4):407.

12. Heaton A, Krudwig K, Lorenson T, Burgess C, Cunningham A, Steinglass R. Doses per vaccine vial container: An understated \& underestimated driver of performance that needs more evidence. Vaccine. 2017 Apr 19;35(17):2272-8.

13. World Health Organization. Training for mid-level managers (MLM).

14. Crocker-Buque T, Mindra G, Duncan R, Mounier-Jack S. Immunization, urbanization and slums-a systematic review of factors and interventions. BMC Public Health. 2017 Dec;17(1):556

15. Hickler B, MacDonald NE, Senouci K, Schuh HB. Efforts to monitor global progress on individual and community demand for immunization: Development of definitions and indicators for the Global Vaccine Action Plan Strategic Objective 2. Vaccine. 2017 Jun 16;35(28):3515-9.

16. Turley R, Saith R, Bhan N, Rehfuess E, Carter B. Slum upgrading strategies involving physical environment and infrastructure interventions and their effects on health and socio-economic outcomes. Cochrane Database Syst Rev. 2012;1.

17. Amin R, De Oliveira TJ, Da Cunha M, Brown TW, Favin M, Cappelier K. Factors limiting immunization coverage in urban Dili, Timor-Leste. Global Health: Science and Practice. 2013 Nov 1;1(3):417-27.

18. Improving immunization in urban slum areas in Nigeria through stakeholder participation: Implication for research. Policy Brief Series 2017, No. 7.

19. Shrestha S, Shrestha M, Wagle RR, Bhandari G. Predictors of incompletion of immunization among children residing in the slums of Kathmandu valley, Nepal: A case-control study. BMC Public Health. 2016 Dec;16(1):970.

20. Cassell JA, Leach M, Fairhead JR, Small M, Mercer $\mathrm{CH}$. The social shaping of childhood vaccination practice in rural and urban Gambia. Health Policy and Planning. 2006 Sep 1;21(5):373-91.

21. Basel PL, Shrestha IB. Factors associated with dropout between bacille Calmette Guerin (BCG) and measles vaccination in a village development committee of a district. J Nepal Health Res Counc. 2012 May 1;10(21):147-51. 